\title{
Article \\ Influence of Isostatic Pressure on the Elastic and Electronic Properties of $\mathrm{K}_{2} \mathrm{SiF}_{6}: \mathrm{Mn}^{4+}$
}

\author{
Mekhrdod Subhoni ${ }^{1,2,3,4, *}$, Umar Zafari ${ }^{3}$, Chong-Geng Ma ${ }^{1}$, Alok M. Srivastava ${ }^{5}$, William W. Beers ${ }^{5}$, \\ William E. Cohen ${ }^{5}\left(\mathbb{D}\right.$, Mikhail G. Brik ${ }^{1,6,7,8, *}$, Michal Piasecki ${ }^{7,9}$ and Tomoyuki Yamamoto $2,10,11$
}

check for

updates

Citation: Subhoni, M.; Zafari, U.; Ma, C.-G.; Srivastava, A.M.; Beers, W.W.; Cohen, W.E.; Brik, M.G.; Piasecki, M.; Yamamoto, T. Influence of Isostatic

Pressure on the Elastic and Electronic Properties of $\mathrm{K}_{2} \mathrm{SiF}_{6}: \mathrm{Mn}^{4+}$. Materials 2022, 15, 613. https://doi.org/ $10.3390 / \mathrm{ma} 15020613$

Academic Editor: Jonas Joos

Received: 10 December 2021

Accepted: 11 January 2022

Published: 14 January 2022

Publisher's Note: MDPI stays neutral with regard to jurisdictional claims in published maps and institutional affiliations.

Copyright: (C) 2022 by the authors. Licensee MDPI, Basel, Switzerland. This article is an open access article distributed under the terms and conditions of the Creative Commons Attribution (CC BY) license (https:// creativecommons.org/licenses/by/ $4.0 /)$.
1 College of Sciences \& CQUPT-BUL Innovation Institute, Chongqing University of Posts and Telecommunications, Chongqing 400065, China; cgma.ustc@gmail.com

2 Kagami Memorial Research Institute for Materials Science and Technology, Waseda University, Tokyo 169-0051, Japan; tymmt@waseda.jp

3 Center of Innovative Development of Science and New Technologies, National Academy of Sciences of Tajikistan, Dushanbe 734025, Tajikistan; zafari_umar@mail.ru

4 Physical Technical Institute, National Academy of Sciences of Tajikistan, Dushanbe 734063, Tajikistan

5 Current Lighting Solutions LLC, 1099 Ivanhoe Road, Cleveland, OH 44110, USA; srivastaam@outlook.com (A.M.S.); william.beers@gecurrent.com (W.W.B.); bill.cohen@gecurrent.com (W.E.C.)

6 Institute of Physics, University of Tartu, W. Ostwald Str. 1, 50411 Tartu, Estonia

7 Faculty of Science and Technology, Jan Długosz University, Armii Krajowej 13/15, PL-42200 Częstochowa, Poland; m.piasecki@ujd.edu.pl

8 Academy of Romanian Scientists, Ilfov Str. No. 3, 050044 Bucharest, Romania

9 Inorganic Chemistry Department, Uzhhorod National University, Pidhirna Str. 46, 88000 Uzhhorod, Ukraine

10 Faculty of Science and Engineering, Waseda University, Tokyo 169-8555, Japan

11 Institute of Condensed-Matter Science, Waseda University, Tokyo 169-8555, Japan

* Correspondence: mehrdodq@gmail.com (M.S.); mikhail.brik@ut.ee (M.G.B.)

\begin{abstract}
Isostatic pressure effects on the elastic and electronic properties of non-doped and $\mathrm{Mn}^{4+}$ doped $\mathrm{K}_{2} \mathrm{SiF}_{6}$ (KSF) have been investigated by first-principles calculations within density functional theory (DFT). Bulk modulus was obtained by the Murnaghan's equation of states (EOS) using the relationship between volume and pressures at pressures between 0 and $40 \mathrm{GPa}$, and elastic constants were calculated by the stress-strain relationship giving small distortions at each pressure point. The other elastic parameters such as shear modulus, sound velocity and Debye temperature, which can be obtained from the elastic constants, were also estimated. The influence of external isostatic pressure on the electronic properties, such as crystal field strength $10 D q$ and emission energy of ${ }^{2} \mathrm{E} \rightarrow$ ${ }^{4} \mathrm{~A}^{2}$ transition $\left(E_{e m}\right)$, of KSF:Mn ${ }^{4+}$ was also studied. The results suggest that $10 D q$ and $E_{e m}$ linearly increase and decrease, respectively, with increasing pressure.
\end{abstract}

Keywords: $\mathrm{K}_{2} \mathrm{SiF}_{6} ; \mathrm{Mn}^{4+}$; isostatic pressure; interionic distances; bulk modulus; elastic constant; Debye temperature; crystal field strength $10 D q$; emission energy

\section{Introduction}

$\mathrm{Mn}^{4+}$-doped phosphors as red photon generating systems have been extensively studied over the past decades due to their great promise for use in LED devices [1-7]. Among a large number of $\mathrm{Mn}^{4+}$ activated red-emitting phosphors, $\mathrm{K}_{2} \mathrm{SiF}_{6}: \mathrm{Mn}^{4+}\left(\mathrm{KSF}: \mathrm{Mn}^{4+}\right)$ has been developed as generators of red photons in phosphor-converted white LEDs (pcLEDs). The sharp-line emission of KSF: $\mathrm{Mn}^{4+}$ peaks at about $630 \mathrm{~nm}$, where the human eye sensitivity to red light is still quite high. With minimum emission beyond $650 \mathrm{~nm}$, the emission spectrum of KSF: $\mathrm{Mn}^{4+}$ is suitable for supplying the red photons necessary to produce white light with high efficacy (lumens per watt) and color rendering index (CRI) in pc-LEDs. Given its commercial importance, detailed analyses of the spectroscopic properties, such as absorption and emission spectra, electronic and geometric properties of $\mathrm{KSF}: \mathrm{Mn}^{4+}$ were performed [8-10] and recently this red phosphor was commercialized [11]. 
The optical properties of the $\mathrm{Mn}^{4+}$ ions (with the $3 \mathrm{~d}^{3}$ electron configuration) are strongly influenced by the composition and crystal structure of the host lattice. In the Tanabe-Sugano diagram for the $\mathrm{d}^{3}$ electron configuration, the " $\mathrm{Mn}^{4+}$-ligand" bonding covalence defines the emission energy of the ${ }^{2} \mathrm{E} \rightarrow{ }^{4} \mathrm{~A}_{2}$ spin-forbidden transition and the strength of the octahedral crystal field denoted as $10 \mathrm{Dq}$. It was determined by DFT calculations that weak $\mathrm{Mn}^{4+}$-ligand hybridization generally leads to higher $\mathrm{Mn}^{4+}$ emission energies [12]. The hybridization between the $\mathrm{Mn}^{4+}$ ions and ligands is related to the $\mathrm{Mn}^{4+}$-ligand distances. The application of pressure is one of the state parameters that can change the peak energy and $10 D q$ by reducing the inter-atomic distances. Studies of the $\mathrm{Mn}^{4+}$ optical properties under external pressure have been reported in the archival literature [13-15]. The goal of this study was to quantitatively explore by first-principles calculations within density functional theory, the electronic, elastic and optical properties of $\mathrm{K}_{2} \mathrm{SiF}_{6}$ in the absence and presence of the activator ion $\left(\mathrm{Mn}^{4+}\right)$.

\section{Computational Method}

All the density functional calculations in this paper were performed by the planewave basis projector augmented wave package, VASP [16], using the generalized gradient approximation proposed by Perdew, Burke and Ernzerhof (GGA-PBE) [17] to express electron-electron correlation. The space group of KSF is Fm-3m with a lattice constant of 8.134 $\AA$ [18], in which the $\mathrm{Si}^{4+}$ ions are surrounded by six $\mathrm{F}^{-}$ions and the local site symmetry is described by the $\mathrm{O}_{\mathrm{h}}$ point group. The $\mathrm{Mn}^{4+}$-doped $\mathrm{KSF}$ models were constructed by replacing one $\mathrm{Si}^{4+}$ ion by one $\mathrm{Mn}^{4+}$ ion in the unit cell of KSF, which includes 36 atoms corresponding to four formula units. After careful convergence tests with respect to the plane wave cut-off energy, $900 \mathrm{eV}$ was selected for the energy cut-off in all the calculations. The Monkhorst-Pack k-points grid sampling [19] was set as $4 \times 4 \times 4$. The structural parameters of non-doped KSF and KSF:Mn ${ }^{4+}$ were optimized by allowing relaxations of the lattice constants and internal atomic positions under isostatic external pressures between 0 and $40 \mathrm{GPa}$ by a 5 GPa step.

\section{Results and Discussion}

The calculated and experimental lattice constants of non-doped KSF and KSF:Mn ${ }^{4+}$ are summarized in Table 1 together with the calculated Si-F and Mn-F bond lengths. The experimental lattice constant of non-doped KSF, $a=8.134 \AA$ [18], is reproduced well by the current calculations, $a=8.336 \AA$, within a typical overestimation due to the GGA-PBE functional use. It is noted here that a slight volume expansion occurs after inclusion of the $\mathrm{Mn}^{4+}$ ions into KSF host. The calculated lattice constant of KSF: $\mathrm{Mn}^{4+}$ is $8.357 \AA$, which is larger than that of KSF $8.336 \AA$. This expansion can be simply explained by the difference in ionic radii of $\mathrm{Si}^{4+}(0.40 \AA)$ and $\mathrm{Mn}^{4+}(0.53 \AA)$. The calculated $\mathrm{Mn}^{4+}-\mathrm{F}^{-}$bond-length is also larger than the calculated $\mathrm{Si}^{4+}-\mathrm{F}^{-}$bond-length by $6.69 \%$, which supports the earlier experimental result of $+7.43 \%$ [20].

Table 1. Comparison of lattice constants, $a$, and bond lengths of $\mathrm{Si}-\mathrm{F}$ and $\mathrm{Mn}-\mathrm{F}$ in the non-doped and $\mathrm{Mn}^{4+}$-doped $\mathrm{K}_{2} \mathrm{SiF}_{6}$, respectively, between experiments and calculations.

\begin{tabular}{|c|c|c|c|}
\hline System & & Calc. (Å) & Exp. (Å) \\
\hline \multirow{2}{*}{$\mathrm{K}_{2} \mathrm{SiF}_{6}$} & $a$ & 8.336 & $8.134^{\mathrm{a}}$ \\
\hline & Si-F & 1.720 & $1.683^{\mathrm{a}}$ \\
\hline \multirow{2}{*}{$\mathrm{K}_{2} \mathrm{SiF}_{6}: \mathrm{Mn}^{4+}$} & $a$ & 8.357 & \\
\hline & $\mathrm{Mn}-\mathrm{F}$ & 1.835 & $1.807^{\mathrm{b}}$ \\
\hline
\end{tabular}

${ }^{\mathrm{a}}$ Ref. [18]. ${ }^{\mathrm{b}}$ Ref. [20]. 
The calculated pressure dependence of the relative volume change $V / V_{0}$ for nondoped KSF is plotted in Figure 1a, which is fitted to the Murnaghan's equation of state (EOS) [21] expressed by:

$$
V / V_{0}=\left(1+P \frac{B^{\prime}}{B}\right)^{-\frac{1}{B^{\prime}}}
$$

where $V$ and $V_{0}$ are volumes at pressure $P$ and ambient pressure, respectively, and $B$ and $B^{\prime}$ are bulk modulus and its pressure derivative, respectively. This fitting yields that $B$ and $B^{\prime}$ are 20.01 GPa and 4.68, respectively. Our results agree well with the earlier study [22] using GGA-PBE, $B=21.79 \mathrm{GPa}$ and $B^{\prime}=4.47$, although a slight difference appears between these two from a difference in calculating methods, i.e., projector augmented wave and pseudopotential methods in the current and earlier [22] studies, respectively. To the best of our knowledge, no experimental bulk modulus for KSF has been reported yet. For $\mathrm{Mn}^{4+}$-doped KSF, $V / V_{0}$ is also plotted in Figure $1 \mathrm{~b}$, which is also fitted to the Murnaghan's EOS. The fitting yields $B=19.84 \mathrm{GPa}$ and $B^{\prime}=4.68$, which implies that inclusion of $\mathrm{Mn}^{4+}$ into KSF leads to a decrease of the bulk modulus.

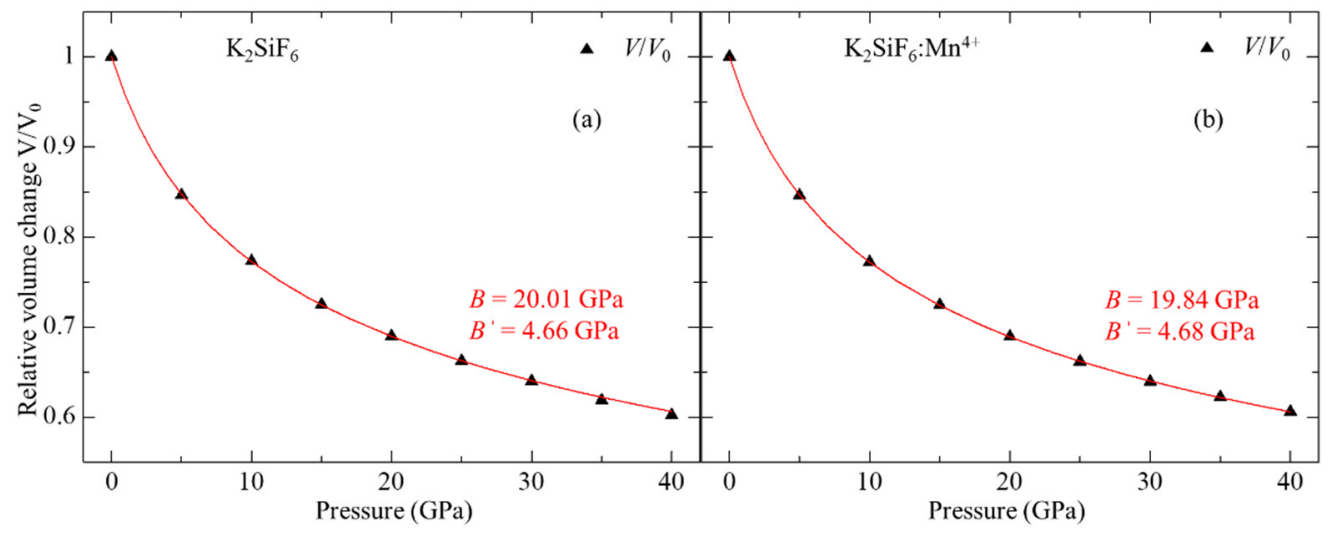

Figure 1. Calculated relative volume $V / V_{0}$ of (a) non-doped and (b) $\mathrm{Mn}^{4+}$-doped $\mathrm{K}_{2} \mathrm{SiF}_{6}$ as a function of pressure. Red solid curves denote fitting results to the Murnaghan's equations of state.

The elastic constants of non-doped and $\mathrm{Mn}^{4+}$-doped KSF were calculated using a stress-strain method [23] implemented in VASP, which are summarized in Table 2 and plotted in Figure 2 as a function of pressure. Here the optimized structure at each pressure was used to calculate the elastic constants. Three irreducible elastic constants for the cubic structure, i.e., $C_{11}, C_{12}$ and $C_{44}$, at zero-pressure were reported in the earlier study [22]; the values are $31.90,9.28$ and $15.10 \mathrm{GPa}$, respectively. Those in the current study show similar values of 28.2, 11.3 and $14.3 \mathrm{GPa}$ for $C_{11}, C_{12}$ and $C_{44}$, respectively. The difference between these two studies is derived from the difference in calculating conditions as discussed above for the bulk modulus. The calculated $C_{11}$ and $C_{12}$ values for the non-doped KSF are larger than those of KSF: $\mathrm{Mn}^{4+}$ at all pressures calculated in this work. On the other hand, the calculated $\mathrm{C}_{44}$ of non-doped KSF is larger than that of $\mathrm{KSF}: \mathrm{Mn}^{4+}$ at zero pressure, the difference between these two becoming smaller as pressure increases, and finally $C_{44}$ of KSF: $\mathrm{Mn}^{4+}$ becomes larger than that of non-doped KSF between 20 and $25 \mathrm{GPa}$. It can be noted that all the elastic constants of both non-doped and KSF:Mn ${ }^{4+}$ increase with increase of pressure, in which $C_{11}$ and $C_{12}$ increase more rapidly than $C_{44}$ as shown in Figure 3. 
Table 2. Pressure dependence of the elastic constants $C_{i j}$ (all in GPa) for the non-doped and $\mathrm{Mn}^{4+}$ doped $\mathrm{K}_{2} \mathrm{SiF}_{6}$.

\begin{tabular}{|c|c|c|c|c|}
\hline System & Pressure & $C_{11}$ & $C_{12}$ & $C_{44}$ \\
\hline \multirow{9}{*}{$\mathrm{K}_{2} \mathrm{SiF}_{6}$} & 0 & 28.2 & 11.3 & 14.3 \\
\hline & 5 & 55.6 & 35.2 & 20.4 \\
\hline & 10 & 77.1 & 52.7 & 30.5 \\
\hline & 15 & 100.1 & 70.5 & 36.6 \\
\hline & 20 & 119.0 & 84.9 & 46.0 \\
\hline & 25 & 137.5 & 99.2 & 52.5 \\
\hline & 30 & 157.4 & 113.3 & 60.1 \\
\hline & 35 & 176.7 & 127.9 & 68.0 \\
\hline & 40 & 195.7 & 141.5 & 75.8 \\
\hline \multirow{9}{*}{$\mathrm{K}_{2} \mathrm{SiF}_{6}: \mathrm{Mn}^{4+}$} & 0 & 23.7 & 9.0 & 11.0 \\
\hline & 5 & 45.8 & 28.1 & 20.1 \\
\hline & 10 & 62.9 & 40.3 & 28.4 \\
\hline & 15 & 90.3 & 62.5 & 36.4 \\
\hline & 20 & 112.0 & 78.3 & 45.0 \\
\hline & 25 & 133.0 & 93.9 & 53.5 \\
\hline & 30 & 154.4 & 109.4 & 61.9 \\
\hline & 35 & 175.0 & 123.7 & 70.5 \\
\hline & 40 & 195.3 & 137.4 & 79.2 \\
\hline
\end{tabular}

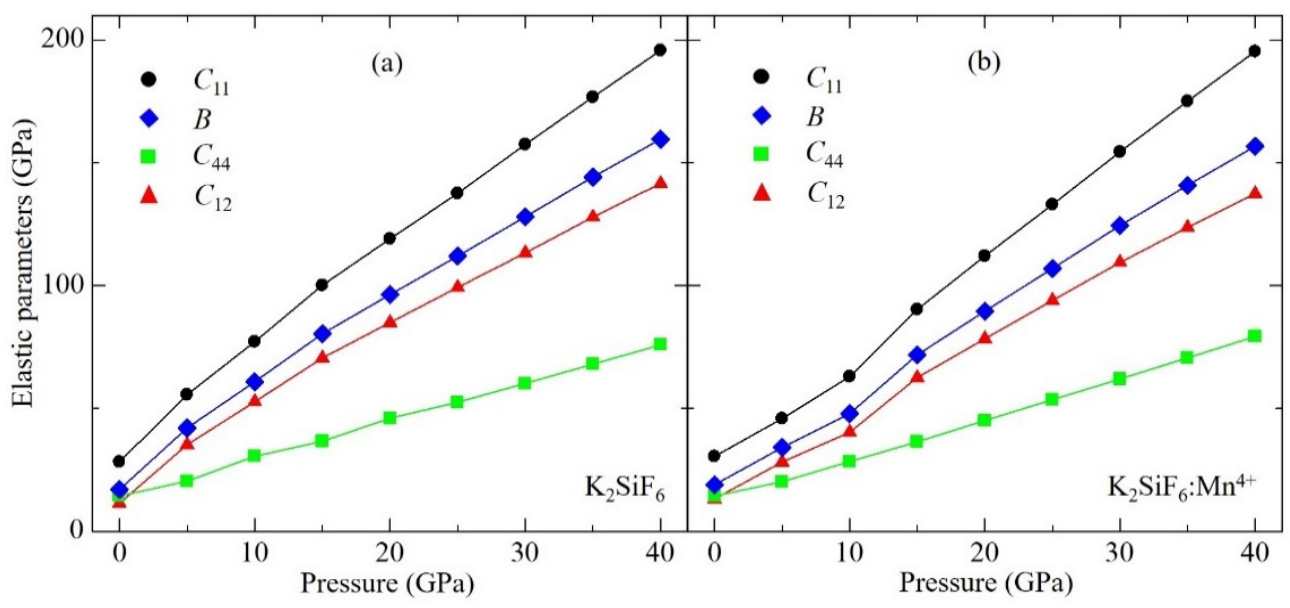

Figure 2. Calculated elastic constants $C_{i j}$ and bulk moduli B for (a) non-doped and (b) $\mathrm{Mn}^{4+}$-doped $\mathrm{K}_{2} \mathrm{SiF}_{6}$ as a function of pressure. 

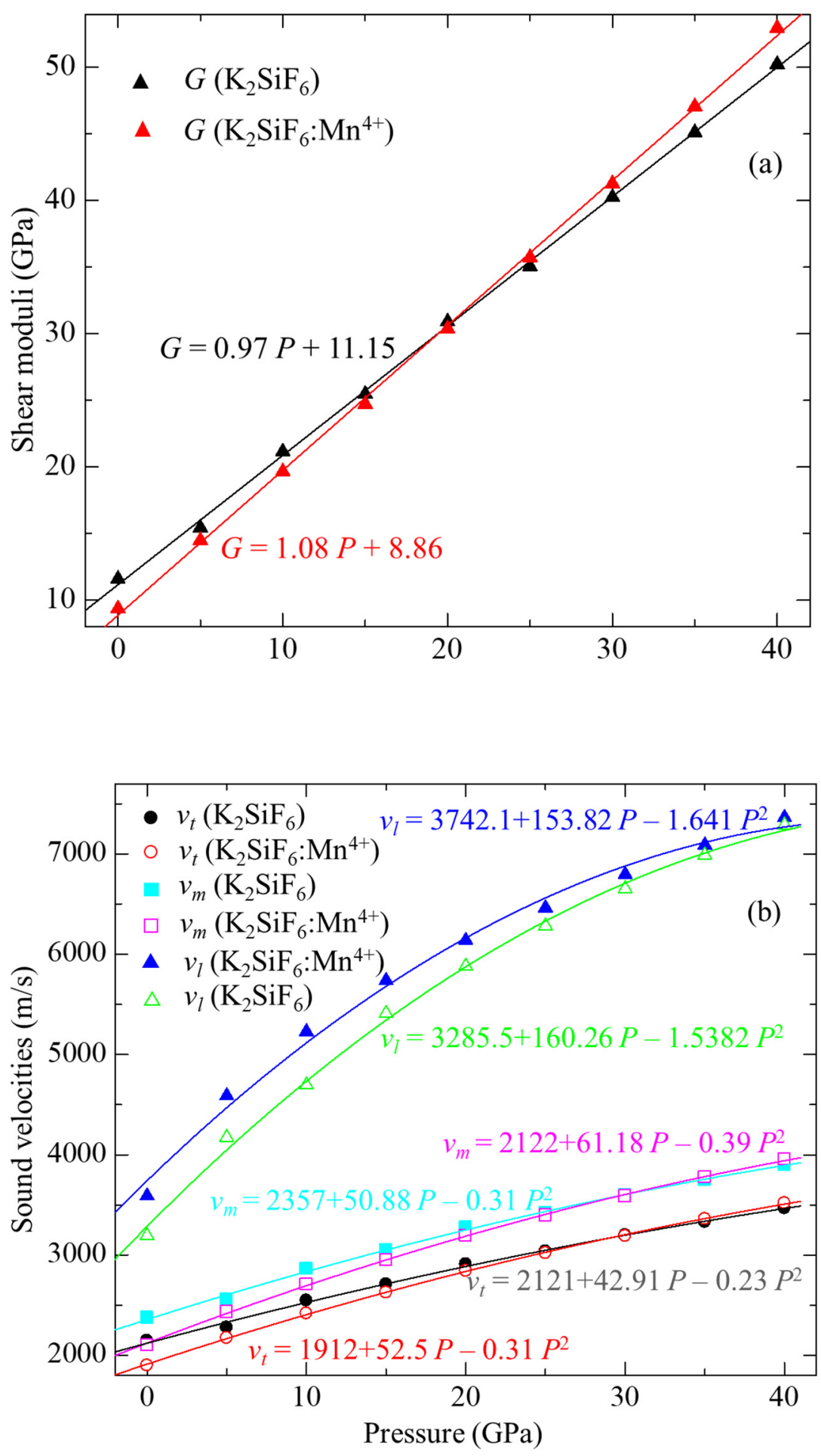

Figure 3. Cont. 


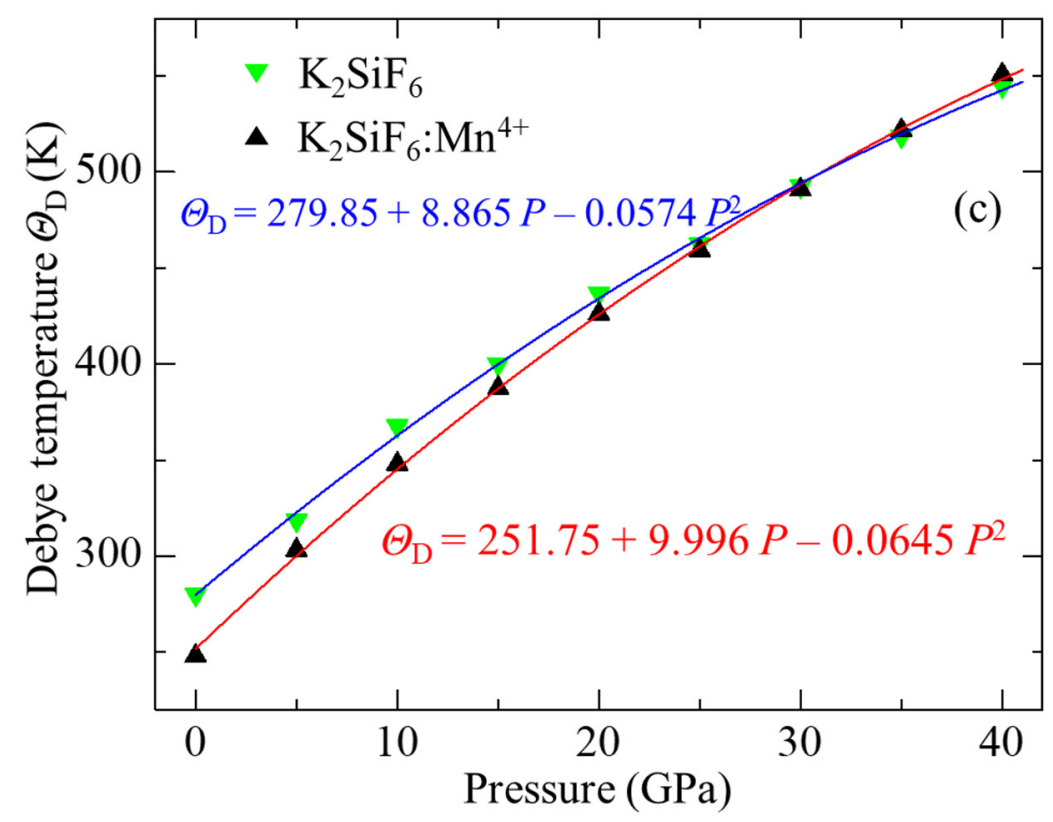

Figure 3. Calculated (a) shear moduli, (b) sound velocities and (c) Debye temperatures for the non-doped and $\mathrm{Mn}^{4+}$-doped $\mathrm{K}_{2} \mathrm{SiF}_{6}$ as a function of pressure.

Using these calculated elastic constants, sound velocities can be obtained. The mean sound velocity $v_{m}$ is expressed [24] in terms of the longitudinal sound velocity $v_{l}$ and the transverse one $v_{t}$ as:

$$
v_{m}=\left[\frac{1}{3}\left(\frac{2}{v_{t}^{3}}+\frac{1}{v_{l}^{3}}\right)\right]^{-1 / 3}
$$

in which $v_{l}$ and $v_{t}$ are calculated [25] by:

$$
v_{l}=\sqrt{\frac{3 B+4 G}{3 \rho}} \text { and } v_{t}=\sqrt{\frac{G}{\rho}}
$$

Here $G=\left(G_{V}+G_{R}\right) / 2$ is the isotropic shear modulus, in which $G_{V}=\left(C_{11}-C_{12}+3 C_{44}\right) / 5$ is the Voight's shear modulus (an upper limit for $G$ ), and $G_{R}=5\left(C_{11}-C_{12}\right) C_{44} /\left[4 C_{44}+3\left(C_{11}-C_{12}\right)\right]$ is the Reuss's shear modulus (a lower limit for $G$ ). Debye temperature, $\Theta_{\mathrm{D}}$, is calculated using the following equation [25]:

$$
\Theta_{D}=\frac{h}{k}\left[\frac{3 n}{4 \pi}\left(\frac{N_{A} \rho}{M}\right)\right]^{1 / 3} v_{m}
$$

where $h$ and $k$ are the Planck's and Boltzmann's constants, respectively, $N_{A}$ is the Avogadro's number, $\rho$ is the density, $M$ is the molecular weight, and $n$ denotes the number of atoms per formula unit (here nine for KSF). The calculated shear modulus, $G$, sound velocities, $v_{m}$, $v_{t}$ and $v_{l}$, and Debye temperature, $\Theta_{\mathrm{D}}$, as a function of pressure for both non-doped and $\mathrm{Mn}^{4+}$-doped KSF are summarized in Table 3 and plotted in Figure $3 \mathrm{a}-\mathrm{c}$ for shear moduli, sound velocities, and Debye temperature, respectively. The $G, v_{m}, v_{t}, v_{l}$ and $\Theta_{\mathrm{D}}$ values for both systems increase with pressure. Inclusion of $\mathrm{Mn}^{4+}$ into KSF leads to a decrease of $G$, $v_{m}, v_{t}, v_{l}$ and $\Theta_{\mathrm{D}}$ at zero pressure. Each of the differences in those parameters between non-doped and $\mathrm{Mn}^{4+}$-doped KSF becomes smaller with increase of pressure. Calculated $G$ of KSF: $\mathrm{Mn}^{4+}$ becomes larger than that of non-doped KSF at pressures between 20 and 25 GPa as in the case of $C_{44}$, while $v_{m}, v_{l}$ and $\Theta_{\mathrm{D}}$ of KSF: $\mathrm{Mn}^{4+}$ becomes larger than that of the non-doped one between 30 and $35 \mathrm{GPa}$. 
Table 3. Calculated shear moduli, sound velocities and Debye temperatures for the non-doped and $\mathrm{Mn}^{4+}$-doped $\mathrm{K}_{2} \mathrm{SiF}_{6}$.

\begin{tabular}{|c|c|c|c|c|c|c|}
\hline System & $P, \mathrm{GPa}$ & $G, \mathrm{GPa}$ & $v_{t}, \mathrm{~m} / \mathrm{s}$ & $v_{l}, \mathrm{~m} / \mathrm{s}$ & $v_{m}, \mathrm{~m} / \mathrm{s}$ & $\Theta_{\mathrm{D}, \mathrm{K}}$ \\
\hline \multirow{9}{*}{$\mathrm{K}_{2} \mathrm{SiF}_{6}$} & 0 & 11.59 & 2146.70 & 3590.80 & 2375.61 & 280 \\
\hline & 5 & 15.44 & 2278.49 & 4589.10 & 2557.08 & 319 \\
\hline & 10 & 21.15 & 2547.46 & 5227.87 & 2861.95 & 368 \\
\hline & 15 & 25.48 & 2708.88 & 5738.69 & 3048.37 & 400 \\
\hline & 20 & 30.91 & 2909.98 & 6138.43 & 3273.96 & 437 \\
\hline & 25 & 35.06 & 3037.20 & 6463.19 & 3418.59 & 462 \\
\hline & 30 & 40.26 & 3199.14 & 6796.39 & 3600.57 & 493 \\
\hline & 35 & 45.13 & 3331.08 & 7089.15 & 3749.39 & 519 \\
\hline & 40 & 50.24 & 3467.45 & 7364.17 & 3902.49 & 545 \\
\hline \multirow{9}{*}{$\mathrm{K}_{2} \mathrm{SiF}_{6}: \mathrm{Mn}^{4+}$} & 0 & 9.36 & 1900.48 & 3193.65 & 2104.06 & 248 \\
\hline & 5 & 14.49 & 2174.34 & 4174.21 & 2432.98 & 303 \\
\hline & 10 & 19.65 & 2419.06 & 4696.07 & 2708.78 & 348 \\
\hline & 15 & 24.72 & 2628.23 & 5410.22 & 2953.19 & 388 \\
\hline & 20 & 30.38 & 2842.32 & 5881.25 & 3194.64 & 426 \\
\hline & 25 & 35.74 & 3020.83 & 6283.09 & 3396.21 & 459 \\
\hline & 30 & 41.28 & 3191.09 & 6653.71 & 3588.09 & 491 \\
\hline & 35 & 47.08 & 3361.47 & 6990.49 & 3779.15 & 522 \\
\hline & 40 & 52.97 & 3518.48 & 7289.33 & 3954.87 & 551 \\
\hline
\end{tabular}

Calculated electronic densities of states (DOSs) of non-doped and $\mathrm{Mn}^{4+}$-doped KSF at 0 GPa are shown in Figure 4. As illustrated in this figure, new orbitals associated with the $\mathrm{Mn}^{4+} 3 \mathrm{~d}$ orbitals appear in the band gap due to a doping of $\mathrm{Mn}^{4+}$ ion. Current results are consistent with our previous report [26], although the previous one was undertaken with larger super cells expanded by $2 \times 1 \times 1$. The $\mathrm{Mn}^{4+}$ ion in KSF is surrounded by six $\mathrm{F}^{-}$ions in a crystal environment of cubic symmetry. Hence, the $3 \mathrm{~d}$ ground-state splits into the triply and doubly degenerated $t_{2 g}$ and $e_{g}$ orbitals, respectively. The3d states of $\mathrm{Mn}^{4+}$ hybridize with the $\mathrm{F}^{-} 2 \mathrm{p}$ states in both $\mathrm{t}_{2 \mathrm{~g}}$ and $\mathrm{e}_{\mathrm{g}}$ orbitals as shown in Figure $4 \mathrm{~b}$. The $\mathrm{Mn}^{4+}$ ion has three $3 d$ electrons, which fully occupy $t_{2 g}$ up-spin state.

To discuss the influence of pressure on the electronic structure, calculated DOSs of KSF: $\mathrm{Mn}^{4+}$ are compared among those at different pressures between 0 and $40 \mathrm{GPa}$, which are shown in Figure 5. The energies of the top of each state originating from $3 \mathrm{~d}$ orbital of $\mathrm{Mn}^{4+}$ ion, i.e., up-spin and down-spin states of $t_{2 g}$ and $e_{g}$, are plotted in Figure 6. As the top of the occupied band was set to zero in Figure 5, the tops of all $t_{2 g}$ up-spin states were located at zero. On the other hand, down-spin $t_{2 g}$ states shift to the lower energy side, while up- and down-spin $e_{g}$ states shift to the higher energy side with increasing pressure. Calculated $10 D q$, which denotes the crystal field strength and is defined as a difference in energy between up-spin $t_{2 g}$ and $e_{g}$ states, at different pressures is summarized in Table 4 and plotted in Figure 7. Calculated $10 D q$ value at zero pressure of $2.76 \mathrm{eV}$ agrees well with the experimental value of $2.74 \mathrm{eV}$ [27], although our current calculations were carried out by GGA-PBE without the effect of strong electron correlation for Mn 3d electrons. As shown in these tables and figures, $10 D q$ increases linearly as pressure increases. Least square fitting to the pressure dependence of $10 D q$ yields $10 D q=0.00346 P+2.734$. In addition, from a geometrical point of view, $10 D q$ values are plotted in Figure 8 as a function of bond length between $\mathrm{Mn}^{4+}$ and sounding $\mathrm{F}^{-}$ions. As shown in this figure, a very good linear relation can be found between $10 \mathrm{Dq}$ and $\mathrm{Mn}-\mathrm{F}$ bond length. The least-square fitting result is $10 D q=-6.37 \mathrm{~d}_{\mathrm{Mn}-\mathrm{F}}+14.46$. This result implies that $10 \mathrm{D} q$ can be estimated by this 
equation for other $\mathrm{Mn}^{4+}$-doped fluorides, in which the doped $\mathrm{Mn}^{4+}$ ion is located at the $\mathrm{O}_{\mathrm{h}}$ symmetry point indicated by six $\mathrm{F}^{-}$ions. This result is consistent with our previous study on $\mathrm{A}_{2} \mathrm{SiF}_{6}$ [26], where $\mathrm{A}=\mathrm{K}, \mathrm{Rb}$ and $\mathrm{Cs}$, in which $10 \mathrm{Dq}$ decreases with increasing $\mathrm{Mn}^{4+}-\mathrm{F}^{-}$bond-length.
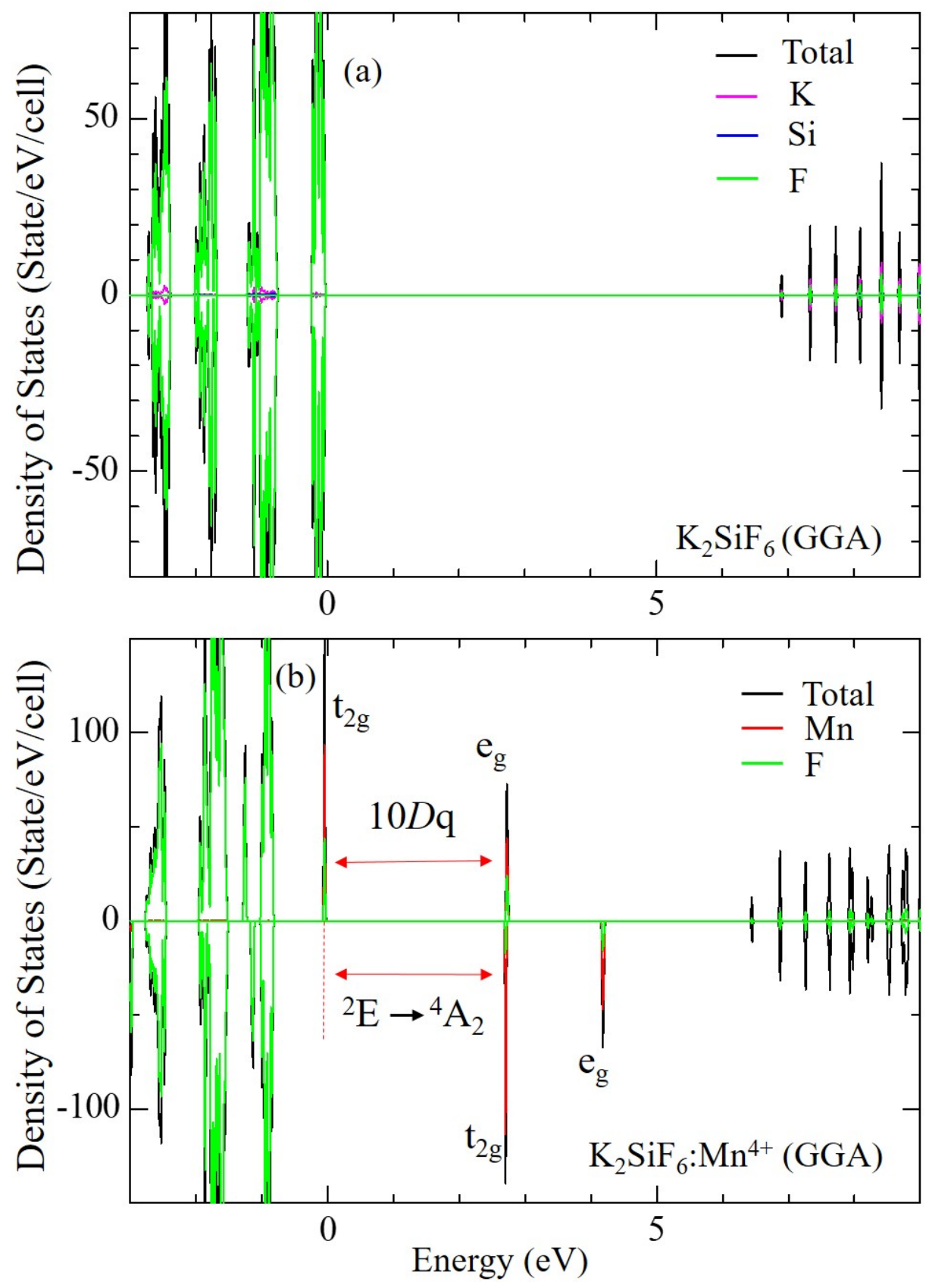

Figure 4. Calculated electronic density of states of (a) non-doped and (b) $\mathrm{Mn}^{4+}$-doped $\mathrm{K}_{2} \mathrm{SiF}_{6}$. 


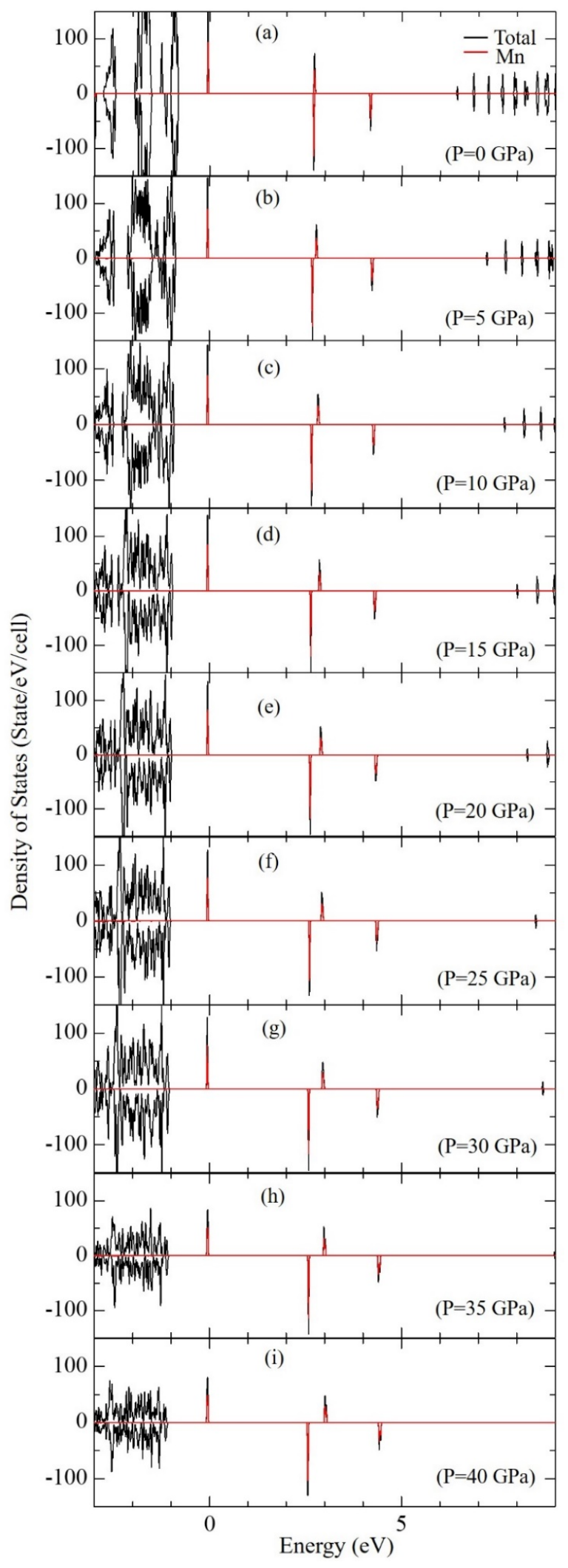

Figure 5. Calculated densities of states (DOSs) of $\mathrm{Mn}^{4+}$-doped $\mathrm{K}_{2} \mathrm{SiF}_{6}$ at $\mathrm{P}=(\mathbf{a}) 0,(\mathbf{b}) 5$, (c) 10 , (d) 15 , (e) 20, (f) 25, (g) 30, (h) 35 and (i) 40 GPa, respectively. 


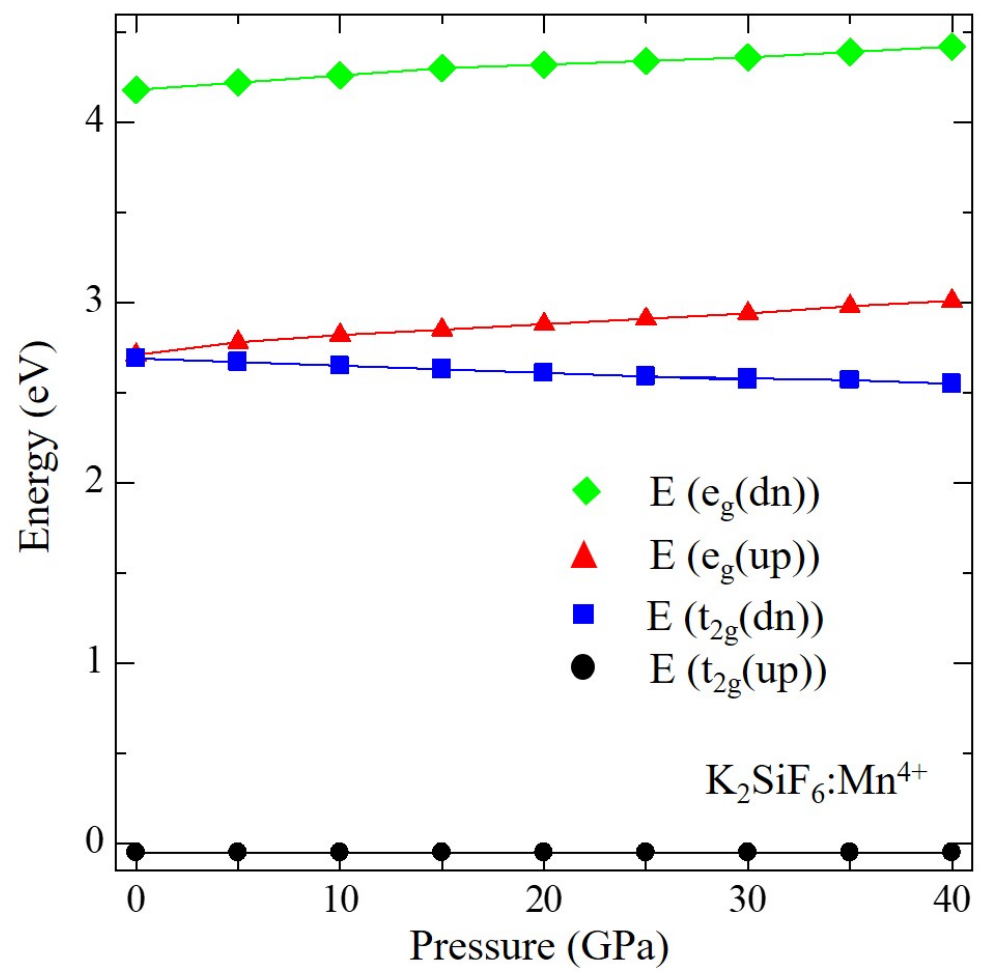

Figure 6. Calculated energy levels of the $t_{2 g}$-up, $t_{2 g}$-down, $e_{g}$-up and $e_{g}$-down states of $\mathrm{Mn}^{4+}$-doped $\mathrm{K}_{2} \mathrm{SiF}_{6}$ as a function of pressure.

Table 4. Comparison of the experimental and calculated ${ }^{2} \mathrm{E} \rightarrow{ }^{4} \mathrm{~A}_{2}$ emission transition energy and $10 D q$ for the $\mathrm{Mn}^{4+}$-doped $\mathrm{K}_{2} \mathrm{SiF}_{6}$ at different pressures.

\begin{tabular}{|c|c|c|c|}
\hline & Pressure (GPa) & Calc. (eV) & Exp. (eV) \\
\hline \multirow{9}{*}{$10 D q$} & 0 & 2.76 & $2.74^{\mathrm{a}}$ \\
\hline & 5 & 2.83 & \\
\hline & 10 & 2.87 & \\
\hline & 15 & 2.90 & \\
\hline & 20 & 2.93 & \\
\hline & 25 & 2.96 & \\
\hline & 30 & 3.00 & \\
\hline & 35 & 3.03 & \\
\hline & 40 & 3.06 & \\
\hline \multirow{9}{*}{${ }^{2} \mathrm{E} \rightarrow{ }^{4} \mathrm{~A}_{2}$} & 0 & 2.74 & $1.99^{b}$ \\
\hline & 5 & 2.72 & \\
\hline & 10 & 2.70 & \\
\hline & 15 & 2.68 & \\
\hline & 20 & 2.66 & \\
\hline & 25 & 2.64 & \\
\hline & 30 & 2.63 & \\
\hline & 35 & 2.62 & \\
\hline & 40 & 2.60 & \\
\hline
\end{tabular}




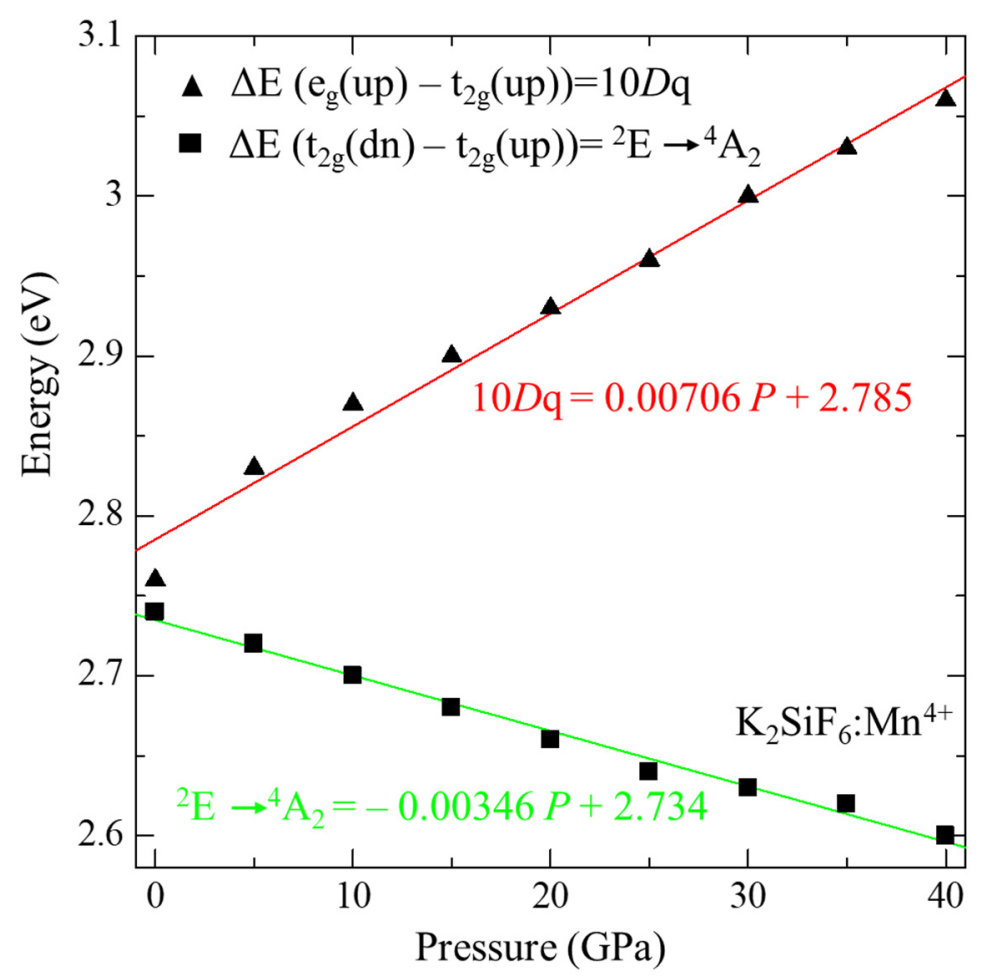

Figure 7. Calculated emission energy, $E_{e m}$, and $10 D q$ for $\mathrm{Mn}^{4+}$-doped $\mathrm{K}_{2} \mathrm{SiF}_{6}$ as a function of pressure. The solid lines are the least-square linear fittings.

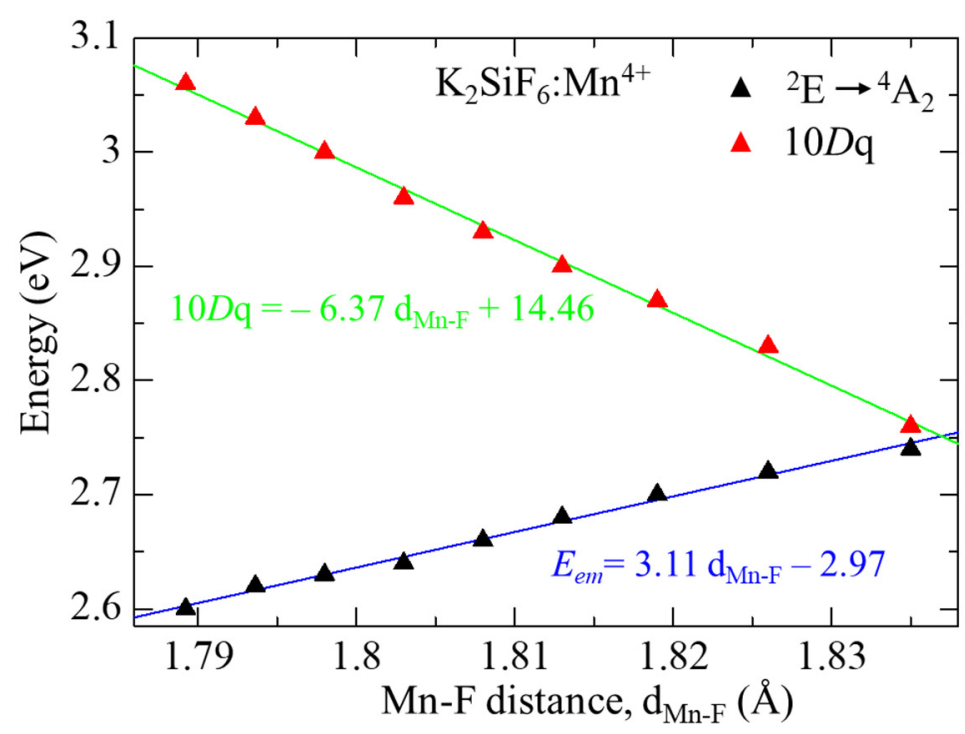

Figure 8. Calculated $10 D q$ parameter and $E_{e m}$ for $\mathrm{K}_{2} \mathrm{SiF}_{6}: \mathrm{Mn}^{4+}$ as a function of $\mathrm{Mn}-\mathrm{F}$ bond length.

The red emission from $\mathrm{Mn}^{4+}$ ion is assigned to the transition from the first excited state ${ }^{2} \mathrm{E}$ to the ground state ${ }^{4} \mathrm{~A}_{2}$ of the $\mathrm{Mn}^{4+} 3 \mathrm{~d}$ state. Electron configuration of the ${ }^{4} \mathrm{~A}_{2}$ state of the $\mathrm{Mn}^{4+}$ ion is all three electrons on up-spin $t_{2 g}$ while that of ${ }^{2} \mathrm{E}$ is two electrons on up-spin $t_{2 g}$ and one on down-spin $t_{2 g}$ states. Here, the transition energy from ${ }^{2} \mathrm{E}$ to ${ }^{4} \mathrm{~A}_{2}$, $E_{e m}$, is calculated by an energy gap between the up-spin and down-spin $t_{2 g}$ states within a one electron approximation to avoid complicated calculations considering multielectron effects to obtain total electronic energy difference between the ${ }^{2} \mathrm{E}$ and ${ }^{4} \mathrm{~A}_{2}$ states. The calculated $E_{e m}$ value at zero pressure is $2.74 \mathrm{eV}$, which is larger than the experimental value of $1.99 \mathrm{eV}$ [9]. This overestimation is derived from the method to obtain $E_{e m}$. However, the change in $E_{e m}$ can be discussed qualitatively as in our earlier report on KSF: $\mathrm{Mn}^{4+}$ [26]. As 
the $\mathrm{d}$ and $\mathrm{f}$ electrons are localized, consideration of a strong electron-electron correlation effect, such as the DFT + U method we have undertaken previously [26], may lead to a better reproduction of $E_{e m}$ than the current calculations with GGA-PBE. In addition, the calculated $E_{e m}$ at zero pressure, i.e., $2.74 \mathrm{eV}$, is larger than that in the previous study on $\mathrm{KSF}: \mathrm{Mn}^{4+}$ [26]. Here, a $1 \times 1 \times 1$ unit cell was used, whereas a $2 \times 1 \times 1$ super cell was employed in our previous study [26], which yield a difference in calculated $E_{e m}$ at zero pressure. $E_{e m}$ as a function of pressure is summarized in Table 4 and plotted in Figure 7. Contrary to the change in $10 D q, E_{e m}$ decreases almost linearly, $E_{e m}=-0.00346 P+2.734$, with increasing pressure. The calculated $E_{e m}$ as a function of $\mathrm{Mn}^{4+}-\mathrm{F}^{-}$bond length is also plotted in Figure 8 for KSF:Mn ${ }^{4+}$. It can be seen that the ${ }^{2} \mathrm{E} \rightarrow{ }^{4} \mathrm{~A}_{2}$ transition energy has very good linear relation with $\mathrm{Mn}-\mathrm{F}$ bond length, $E_{\text {em }}=3.11 \mathrm{~d}_{\mathrm{Mn}-\mathrm{F}}-2.97$.

\section{Conclusions}

First-principles calculations have been carried out for the non-doped and $\mathrm{Mn}^{4+}$-doped $\mathrm{K}_{2} \mathrm{SiF}_{6}$ to study the influence of isostatic pressure on the geometric structure, elastic and electronic properties of $\mathrm{K}_{2} \mathrm{SiF}_{6}: \mathrm{Mn}^{4+}$ red phosphor. In particular, the pressure effect on the elastic properties such as elastic constants, shear modulus, sound velocity and Debye temperature between non-doped and $\mathrm{Mn}^{4+}$-doped systems was discussed. Pressure dependence of two important electronic parameters, i.e., $10 D q$ and the emission energy of the ${ }^{2} \mathrm{E} \rightarrow{ }^{4} \mathrm{~A}_{2}$ transition, of $\mathrm{KSF}: \mathrm{Mn}^{4+}$ have been investigated, which yield good linear relationships both between $10 \mathrm{Dq}$ and external pressure and between emission energy of ${ }^{2} \mathrm{E}$ $\rightarrow{ }^{4} \mathrm{~A}_{2}$ and pressure. It can be noted that the current analysis of the pressure effect on $10 \mathrm{Dq}$ and emission energy of the ${ }^{2} \mathrm{E} \rightarrow{ }^{4} \mathrm{~A}_{2}$ transition provided empirical formulae to obtain these values as a function of bond-length between $\mathrm{Mn}^{4+}$ and surrounding $\mathrm{F}^{-}$ions, which can be used for the estimation of these important parameters for other Mn-doped fluorides.

Author Contributions: Conceptualization, T.Y., M.G.B. and M.S.; methodology, M.S. and U.Z.; software, M.S., U.Z. and T.Y.; validation, T.Y., C.-G.M., M.P., M.G.B., W.W.B., W.E.C. and A.M.S.; formal analysis, M.S., U.Z., T.Y., M.G.B., M.P. and C.-G.M.; investigation, M.S., U.Z. and T.Y.; resources, M.G.B.; data curation, M.S., T.Y., C.-G.M. and M.P.; writing-original draft preparation, M.S., T.Y., M.G.B. and A.M.S.; writing-review and editing, T.Y., M.G.B., A.M.S., W.W.B. and W.E.C.; visualization, M.S. and U.Z.; supervision, T.Y., M.G.B. and A.M.S.; project administration, T.Y. and M.G.B.; funding acquisition, T.Y. and M.G.B. All authors have read and agreed to the published version of the manuscript.

Funding: This work was partly carried out at the Joint Research Center for Environmentally Conscious Technologies in Materials Science (project No. 30009, 30012, 31008, 31017, 02018, 02021, 02022, 02115, 02116 and 02117) at ZAIKEN, Waseda University, and supported by JSPS KAKENHI Grant number 20K05065. M.S. and U.Z. thank for support the IICN under the CIS Grant number 21-109 and 21-107, respectively. The authors are grateful to Kholmirzo Kholmurodov for his continuous encouragement and support. M.G.B. appreciates the supports from the Program for the Foreign Experts (Grant No. W2017011) offered by Chongqing University of Posts and Telecommunications and the National Foreign Experts Program for "Belt and Road Initiative" Innovative Talent Exchange (Grant No. DL2021035001L), Estonian Research Council grant PUT PRG111, European Regional Development Fund (TK141), and NCN project 2018/31/B/ST4/00924. C.-G. Ma acknowledges the support of the National Natural Science Foundation of China (Grant No. 52161135110) and ChinaPoland Intergovernmental Science and Technology Cooperation Program (Grant No. 2020[15]/10). M.S. appreciates the support from 2021 Chongqing Postdoctoral International Exchange Program of China Postdoctoral Science Foundation.

Institutional Review Board Statement: Not applicable.

Informed Consent Statement: Not applicable.

Data Availability Statement: The raw/processed data required to reproduce these findings cannot be shared at this time as the data also form a part of an ongoing study. 
Conflicts of Interest: The authors declare no conflict of interest. The funders had no role in the design of the study; in the collection, analyses, or interpretation of data; in the writing of the manuscript, or in the decision to publish the results.

\section{References}

1. Brik, M.; Srivastava, A. On the optical properties of the $\mathrm{Mn}^{4+}$ ion in solids. J. Lumin. 2013, 133, 69-72. [CrossRef]

2. Arai, Y.; Adachi, S. Optical properties of $\mathrm{Mn}^{4+}$-activated $\mathrm{Na}_{2} \mathrm{SnF}_{6}$ and $\mathrm{Cs}_{2} \mathrm{SnF}_{6}$ red phosphors. J. Lumin. 2011, 131, 2652-2660. [CrossRef]

3. Srivastava, A.; Brik, M. Ab initio and crystal field studies of the $\mathrm{Mn}^{4+}$-doped $\mathrm{Ba}_{2} \mathrm{LaNbO}_{6}$ double-perovskite. J. Lumin. 2012, 132, 579-584. [CrossRef]

4. Nguyen, H.-D.; Liu, R.-S. Narrow-band red-emitting $\mathrm{Mn}^{4+}$-doped hexafluoride phosphors: Synthesis, optoelectronic properties, and applications in white light-emitting diodes. J. Mater. Chem. C 2016, 4, 10759-10775. [CrossRef]

5. Adachi, S. Photoluminescence properties of $\mathrm{Mn}^{4+}$-activated oxide phosphors for use in white-LED applications: A review. $J$. Lumin. 2018, 202, 263-281. [CrossRef]

6. Adachi, S. Crystal-field and Racah parameters of $\mathrm{Mn}^{4+}$ ion in red and deep red-emitting phosphors: Fluoride versus oxide phosphor. J. Lumin. 2020, 218, 116829. [CrossRef]

7. Senden, T.; Van Dijk-Moes, R.J.A.; Meijerink, A. Quenching of the red $\mathrm{Mn}^{4+}$ luminescence in $\mathrm{Mn}^{4+}$-doped fluoride LED phosphors. Light Sci. Appl. 2018, 7, 8. [CrossRef]

8. Adachi, S. Photoluminescence spectra and modeling analyses of $\mathrm{Mn}^{4+}$-activated fluoride phosphors: A review. J. Lumin. 2018, 197, 119-130. [CrossRef]

9. Arai, T.; Adachi, S. Excited States of $3 \mathrm{~d} 33$ Electrons in $\mathrm{K}_{22} \mathrm{SiF}_{66}: \mathrm{Mn}^{4+4+}$ Red Phosphor Studied by Photoluminescence Excitation Spectroscopy. Jpn. J. Appl. Phys. 2011, 50, 092401. [CrossRef]

10. Sijbom, H.F.; Joos, J.; Martin, L.; Eeckhout, K.V.D.; Poelman, D.; Smet, P. Luminescent Behavior of the $\mathrm{K}_{2} \mathrm{SiF}_{6}: \mathrm{Mn}^{4+} \mathrm{Red} \mathrm{Phosphor}$ at High Fluxes and at the Microscopic Level. ECS J. Solid State Sci. Technol. 2015, 5, R3040-R3048. [CrossRef]

11. Setlur, A.A.; Radkov, E.V.; Henderson, C.S.; Her, J.-H.; Srivastava, A.M.; Karkada, N.; Kishore, M.S.; Kumar, N.P.; Aesram, D.; Deshpande, A.; et al. Energy-Efficient, High-Color-Rendering LED Lamps Using Oxyfluoride and Fluoride Phosphors. Chem. Mater. 2010, 22, 4076-4082. [CrossRef]

12. Du, M.H. Chemical trends of $\mathrm{Mn}^{4+}$ emission in solids. J. Mater. Chem. C 2014, 2, 2475-2481. [CrossRef]

13. Wang, Y.; Wen, T.; Tang, L.; Yang, L.; Yang, W.; Zhao, Y. Impact of hydrostatic pressure on the crystal structure and photoluminescence properties of $\mathrm{Mn}^{4+}$-doped $\mathrm{BaTiF}_{6}$ red phosphor. Dalton Trans. 2015, 44, 7578-7585. [CrossRef] [PubMed]

14. Zhang, N.; Tsai, Y.-T.; Fang, M.-H.; Ma, C.-G.; Lazarowska, A.; Mahlik, S.; Grinberg, M.; Chiang, C.-Y.; Zhou, W.; Lin, J.G.; et al. Aluminate Red Phosphor in Light-Emitting Diodes: Theoretical Calculations, Charge Varieties, and High-Pressure Luminescence Analysis. ACS Appl. Mater. Interfaces 2017, 9, 23995-24004. [CrossRef]

15. Lazarowska, A.; Mahlik, S.; Grinberg, M.; Lin, C.C.; Liu, R.-S. Pressure effect on the zero-phonon line emission of Mn ${ }^{4+}$ in K2SiF6. J. Chem. Phys. 2015, 143, 134704. [CrossRef]

16. Kresse, G.; Furthmüller, J. Efficiency of ab-initio total energy calculations for metals and semiconductors using a plane-wave basis set. Comput. Mater. Sci. 1996, 6, 15-50. [CrossRef]

17. Perdew, J.P.; Burke, K.; Wang, Y. Generalized gradient approximation for the exchange-correlation hole of a many-electron system. Phys. Rev. B 2014, 54, 16533-16539. [CrossRef]

18. Loehlin, J.H. Redetermination of the Structure of Potassium Hexafluorosilicate, $\mathrm{K}_{2} \mathrm{SiF}_{6}$. Acta Cryst. C 1984, 40, 570. [CrossRef]

19. Monkhorst, H.J.; Pack, J.D. Special points for Brillouin-zone integrations. Phys. Rev. B 1976, 13, 5188. [CrossRef]

20. Novita, M.; Honma, T.; Hong, B.; Ohishi, A.; Ogasawara, K. Study of multiplet structures of $\mathrm{Mn}^{4+}$ activated in fluoride crystals. J. Lumin. 2016, 169, 594-600. [CrossRef]

21. Murnaghan, F.D. The Compressibility of Media under Extreme Pressures. Proc. Natl. Acad. Sci. USA 1944, 30, 244-247. [CrossRef]

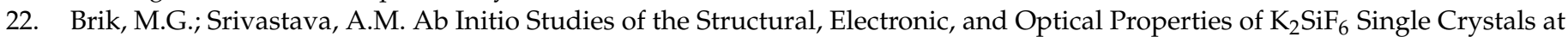
Ambient and Elevated Hydrostatic Pressure. J. Electrochem. Soc. 2012, 159, J212-J216. [CrossRef]

23. Nielsen, O.H.; Martin, R.M. First-Principles Calculation of Stress. Phys. Rev. Lett. 1983, 50, 697-700. [CrossRef]

24. Poirier, J.; Brown, J.M. Introduction to the Physics of the Earth's Deep Interior. Phys. Today 1992, 45, 66-67. [CrossRef]

25. Anderson, O.L. A simplified method for calculating the debye temperature from elastic constants. J. Phys. Chem. Solids 1963, 24, 909-917. [CrossRef]

26. Subhoni, M.; Zafari, U.; Srivastava, A.M.; Beers, W.W.; Cohen, W.; Brik, M.G.; Yamamoto, T. First-principles investigations of geometrical and electronic structures of $\mathrm{Mn}^{4+}$ doped $\mathrm{A}_{2} \mathrm{SiF}_{6}(\mathrm{~A}=\mathrm{K}, \mathrm{Rb}, \mathrm{Cs})$ red phosphors. Opt. Mater. 2021, 115, 110986. [CrossRef]

27. Paulusz, A.G. Efficient Mn(IV) Emission in Fluorine Coordination. J. Electrochem. Soc. 1973, 120, 942. [CrossRef] 